\title{
Pycnogenol attenuates atherosclerosis by regulating lipid metabolism through the TLR4-NF-kB pathway
}

\begin{abstract}
Hong Luo ${ }^{1,3}$, Jing Wang ${ }^{2,3}$, Chenhui Qiao ${ }^{1}$, Ning Ma ${ }^{1}$, Donghai Liu ${ }^{1}$ and Weihua Zhang ${ }^{1}$
Atherosclerosis is a leading cause of death worldwide and is characterized by lipid-laden foam cell formation. Recently, pycnogenol (PYC) has drawn much attention because of its prominent effect on cardiovascular disease (CVD). However, its protective effect against atherosclerosis and the underlying mechanism remains undefined. Here PYC treatment reduced areas of plaque and lipid deposition in atherosclerotic mice, concomitant with decreases in total cholesterol and triglyceride levels and increases in HDL cholesterol levels, indicating a potential antiatherosclerotic effect of PYC through the regulation of lipid levels. Additionally, PYC preconditioning markedly decreased foam cell formation and lipid accumulation in lipopolysaccharide (LPS)-stimulated human THP-1 monocytes. A mechanistic analysis indicated that PYC decreased the lipid-related protein expression of adipose differentiation-related protein (ADRP) and adipocyte lipid-binding protein (ALBP/aP2) in a dose-dependent manner. Further analysis confirmed that PYC attenuated LPS-induced lipid droplet formation via ADRP and ALBP expression through the Toll-like receptor 4 (TLR4) and nuclear factor-кB (NF-kB) pathway, because pretreatment with anti-TLR4 antibody or a specific inhibitor of NF-KB (PDTC) strikingly mitigated the LPS-induced increase in ADRP and ALBP. Together, our results provide insight into the ability of PYC to attenuate bacterial infection-triggered pathological processes associated with atherosclerosis. Thus PYC may be a potential lead compound for the future development of antiatherosclerotic CVD therapy. Experimental \& Molecular Medicine (2015) 47, e191; doi:10.1038/emm.2015.74; published online 23 October 2015
\end{abstract}

\section{INTRODUCTION}

Atherosclerosis and the subsequent cardiovascular complications, such as myocardial infarction, coronary atherosclerosis and stroke, are leading causes of death worldwide. ${ }^{1}$ It is widely accepted that atherosclerosis is a complex chronic inflammatory disease that is characterized by the accumulation of lipid droplets and fibrous elements in arteries. ${ }^{2}$

The natural plant compounds known as flavonoids have recently been found to have anti-inflammatory, antithrombotic and protective cardiovascular properties., ${ }^{3,4}$ One of these compounds, pycnogenol (PYC), has drawn increasing attention because of its antioxidative, anti-inflammatory and antiplatelet effects. Pycnogenol is a natural extract of the French maritime pine tree (Pinus maritima) and has multiple biological and pharmacological activities in many diseases, including cardiovascular diseases (CVDs) and their complications. ${ }^{5,6}$ Endothelial dysfunction is known to be a precursor of atherosclerosis and cardiovascular events. ${ }^{7-9}$ It has been demonstrated that PYC can improve endothelial function in patients with coronary artery disease, by abrogating oxidative stress. ${ }^{10} \mathrm{~A}$ clinical analysis has indicated that PYC supplementation effectively reduces CVD risk factors in subjects with type 2 diabetes. ${ }^{1}$ However, the protective antiatherosclerotic properties of PYC and the precise underlying molecular mechanism remain unclear.

Lipid droplets are the main hallmark of atherosclerosis, followed by the formation of the earliest detected lesion, the fatty streak. During this process, macrophages are activated by Toll-like receptor (TLR) ligands such as lipopolysaccharide (LPS) and then take up oxidized low-density lipoprotein (ox-LDL) to form foam cells, triggering atherogenic progression. ${ }^{11}$ Blocking lipid deposition notably downregulates atherosclerotic coronary lesion formation, indicating that dampening lipid levels will be potential targets for atherosclerosis and cardiovascular events. ${ }^{2,12}$ It has been confirmed that various proteins are present on the surface of lipid droplets and have essential roles in the synthesis, storage and degradation of lipids. ${ }^{13}$ For example, adipose differentiation-related protein (ADRP) has been shown to be dramatically upregulated in macrophage foam cells at the atherosclerotic lesion.

\footnotetext{
${ }^{1}$ Department of Cardiovascular Surgery, The First Affiliated Hospital of Zhengzhou University, Henan, China and ${ }^{2}$ Department of Stomatology, The First Affiliated Hospital of Zhengzhou University, Henan, China

${ }^{3}$ These authors contributed equally to this work and are considered co-first authors.

Correspondence: Dr H Luo, Department of Cardiovascular Surgery, The First Affiliated Hospital of Zhengzhou University, No. 1, Jianshe East Road, Henan 453000, China. 
Following stimulation with ADRP, obvious lipid accumulation and lipid droplet formation is detected. ${ }^{14}$ The absence of ADRP strikingly attenuates foam cell formation, resulting in the amelioration of atherosclerosis. ${ }^{15}$ Adipocyte lipid-binding protein (ALBP/aP2), also known as AFBP4, is a major lipid droplet protein and is markedly upregulated in LPS-induced macrophage foam cells. ${ }^{16,17}$ Previous studies have shown that both of these proteins facilitate foam cell formation, and their absence provides protection against atherosclerosis. ${ }^{15,17}$

It has been reported that PYC dramatically antagonizes TLR-4-mediated ADRP expression in macrophages. ${ }^{18}$ Therefore, we hypothesized that PYC might have beneficial antiatherosclerotic effects by regulating lipid-laden foam cell formation via mediation of lipid-related protein expression. The purpose of this study was to investigate the effect of PYC on the progression of atherosclerosis and the underlying mechanism. The present findings provide insight into the ability of PYC to attenuate the bacterial infection-triggered pathological process of atherosclerosis.

\section{MATERIALS AND METHODS}

\section{Reagents and antibodies}

Unless mentioned otherwise, all of the reagents were obtained from Sigma-Aldrich (St Louis, MO, USA). PYC was provided by Tradepia Co. (Kasukabe, Saitama, Japan). The NF-кB inhibitor PDTC was from Calbiochem (La Jolla, CA, USA). The polyclonal antibodies against human NF- $\kappa \mathrm{B}$ p65 and ADRP were from Fitzgerald Industries Intl. (Concord, MA, USA). Mouse anti-human ALBP antibody was obtained from Biotang Inc. (Waltham, MA, USA). Rabbit antihuman TLR4 antibody was purchased from Santa Cruz Biotechnology (Santa Cruz, CA, USA). Peroxidase-conjugated goat anti-mouse and anti-rabbit antibodies were from Dako Cytomation (Glostrup, Denmark) and Vector Laboratories, Inc. (Burlingame, CA, USA), respectively. Monoclonal rat anti-mouse MOMA-2 antibody was from Dianova (Augst, Switzerland).

\section{Experiment model}

Thirty-two male apolipoprotein E (ApoE)-deficient mice (3 weeks old, 25-30 g) were obtained from Changzhou Cavens Laboratory Animal Co., LTD (Changzhou, China) and were maintained under standard room temperature, dark-light cycle and humidity conditions. After allowing a week for acclimatization, all of the mice were randomly divided into two groups. Approximately $2 \mathrm{mg} \mathrm{ml}^{-1}$ LPS was injected three times a week to induce acute atherosclerosis, together with a high-fat diet (20\% fat, 20\% sugar and $1.25 \%$ cholesterol), for 12 weeks. PYC $\left(0.15 \mathrm{mg} \mathrm{kg}^{-1}\right)$ was administered orally once a day after LPS treatment. All of the experiments were conducted under a protocol that had been approved by the Institutional Animal Care and Use Committee of Xinxiang Medical University. All efforts were made to minimize suffering.

\section{Histological evaluation}

After being anesthetized with pentobarbital at 12 weeks, the animals were perfused with cold phosphate-buffered saline (PBS) followed by cold buffered formalin. Aorta specimens were collected and fixed with formalin. Following dehydration, the specimens were embedded in paraffin. For histology, serial, $4-\mu \mathrm{m}$-thick sections were obtained from each specimen and were then stained for routine histology (hematoxylin/eosin, H\&E) and lipids levels (Oil Red O). Subsequently, high-resolution images were obtained with an Olympus BX-40 microscope or a Zeiss LSM5 Meta confocal microscope (Zeiss, Jena, Germany). Necrotic cores were assessed based on H\&E and quantified by planimetry (Diskus software; Hilgers, Bonn). To calculate the lipid content relative to the plaque area, all of the images were analyzed after digitization using the automated image analysis software (Clemex Technologies Inc., Montreal, Quebec, Canada).

\section{Immunohistochemistry}

To detect macrophages in plaques, immunohistochemical staining was used. Briefly, the slices were cultured with rat anti-mouse Monocyte/ Macrophage Marker Antibody (MOMA-2) antibodies (1:1000) at $4{ }^{\circ} \mathrm{C}$ overnight. After three washes with Tris-buffered saline with Tween (TBST), biotin-conjugated rabbit anti-rat IgG was introduced as a secondary antibody for another $1 \mathrm{~h}$. The color development was analyzed using a universal streptavidin/biotin immunoperoxidase detection system (Thermo Shandon, Pittsburgh, PA, USA) according to the manufacturer's instructions. Next, the slides were placed on the ACIS automated imaging system (DAKO, Carpinteria, CA, USA) to quantify the tissue staining.

\section{Lipid analysis and lipoprotein profile measurement}

Approximately 12 weeks later, the mice were killed via an injection of pentobarbital $\left(200 \mathrm{mg} \mathrm{kg}^{-1}\right)$. Blood samples were collected from mouse orbital veins. The serum levels of total cholesterol (TC), triglycerides, HDL cholesterol (LDL-C) and HDL cholesterol (HDL-C) were measured via a microplate method using the Beckman Coulter Synchon Cholesterol 300 reagent (Beckman Coulter, Inc., Brea, CA, USA). Plasma lipoprotein fractions were evaluated by fast performance liquid chromatography gel filtration coupled with two Superose6 HR 10/30 columns (Amersham Pharmacia Biotech Inc., Piscataway, NJ, USA). A commercially available kit (Shanghai Hushang Biotechnology Co. Ltd., Shanghai, China) and an automatic biochemistry analyzer (Hitachi, Tokyo, Japan) were also used for the analysis.

\section{Preparation of ox-LDL}

The ox-LDL was prepared as previously described. ${ }^{19}$ Briefly, $200 \mu \mathrm{g} \mathrm{ml}^{-1} \mathrm{LDL}$ was introduced into $20 \mu \mathrm{M} \mathrm{CuSO}_{4}$ in PBS for oxidation at $37^{\circ} \mathrm{C}$. Approximately $24 \mathrm{~h}$ later, $40 \mu \mathrm{M}$ butylhydroxytoluene in ethanol was used to stop the oxidative reactions. Ox-LDL was separated from the preparation by dialysis against PBS for $24 \mathrm{~h}$, followed by fluorometric confirmation (excitation wavelength, $515 \mathrm{~nm}$; emission wavelength, $553 \mathrm{~nm}$ ) by measurement of thiobarbituric acid-reactive substances.

\section{Cell culture and treatments}

The human monocytic leukemia cell line THP-1 was obtained from the American Type Culture Collection (ATCC, Manassas, VA, USA). The cells were maintained in RPMI 1640 medium containing $10 \%$ fetal bovine serum, $100 \mu \mathrm{g} \mathrm{ml}^{-1}$ streptomycin and penicillin at $37^{\circ} \mathrm{C}$ with $5 \% \mathrm{CO}_{2}$. PYC was dissolved in dimethyl sulfoxide to a final of $1 \%$ concentration before use. Before stimulation with the indicated dose of PYC for $8 \mathrm{~h}$, THP-1 monocytes were preconditioned with anti-TLR4 antibody $\left(10 \mu \mathrm{g} \mathrm{ml}^{-1}\right)$ or $25 \mu \mathrm{M}$ PDTC for $2 \mathrm{~h}$. After these treatments, the cells were stimulated with $10 \mu \mathrm{g} \mathrm{ml}^{-1}$ LPS prior to incubation with CuoxLDL. 

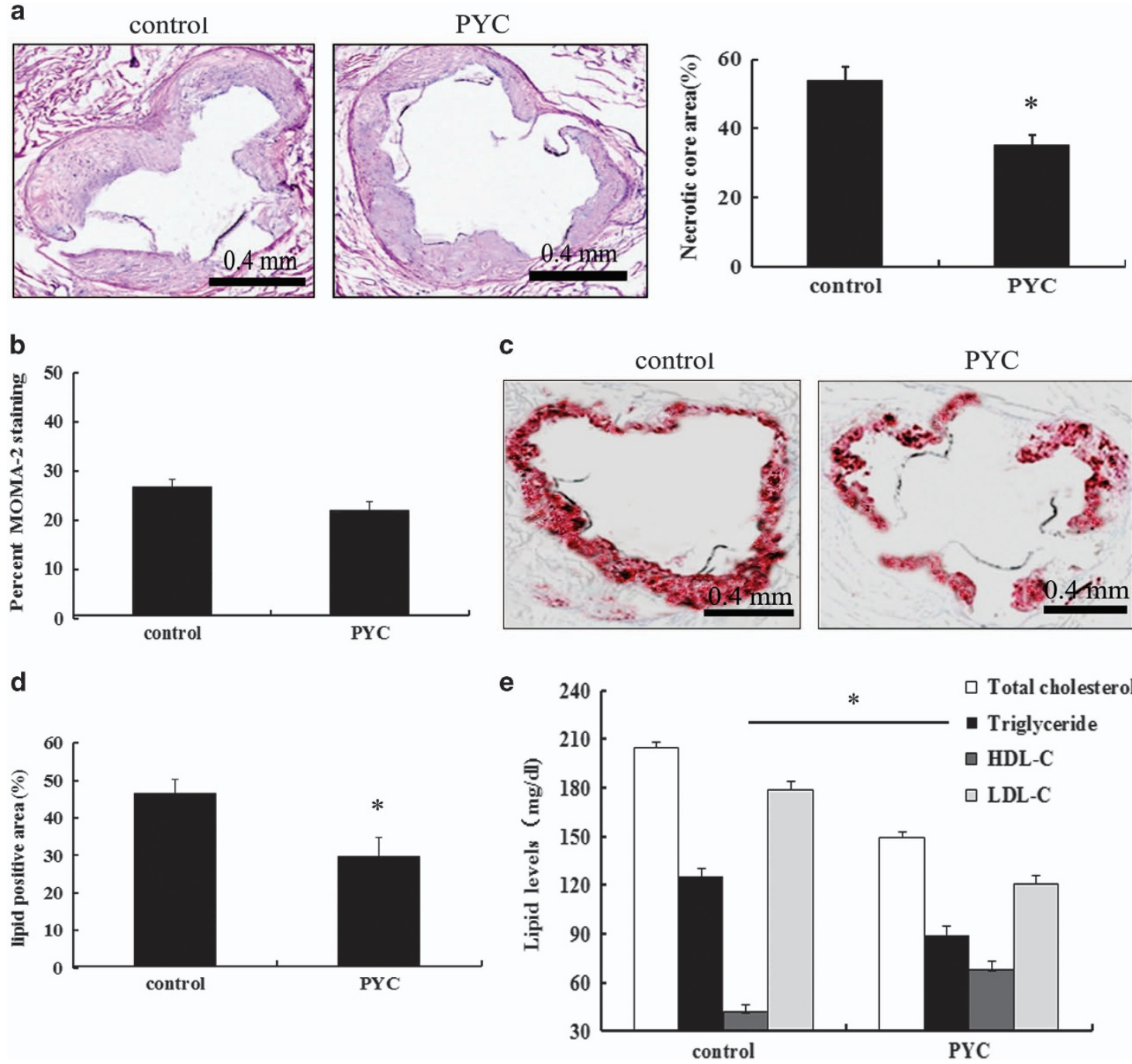

Figure 1 Effects of PYC on atherosclerotic plaques and lipid levels. Following the construction of an atherosclerotic ApoE-/- mouse model induced by LPS and a high-fat diet, PYC $\left(0.15 \mathrm{mg} \mathrm{kg}^{-1}\right)$ was administered orally once a day after LPS treatment. Twelve weeks later, sections of the aorta were analyzed by H\&E staining (a). Immunohistochemical analysis of macrophage antigen (MOMA)-2 was performed to evaluate the macrophage numbers (b). Oil Red $\mathrm{O}$ was used to examine the lipid accumulation in plaques (c, $\mathbf{d}$ ). The serum lipid levels were also measured, including total cholesterol, triglycerides, LDL cholesterol (LDL-C) and HDL cholesterol (HDL-C) (e). $* P<0.05$

\section{Oil Red O staining}

After cultivation with CuoxLDL for $10 \mathrm{~h}$, the cells were washed three times with PBS followed by fixation with $10 \%$ formalin. To highlight the characteristic lipid accumulation in macrophage-derived foam cells, the cells were stained for $30 \mathrm{~min}$ at $37^{\circ} \mathrm{C}$ in freshly diluted Oil Red O solution (Sigma, St Louis, MO, USA) followed by three rinses with water. The cell nuclei were then stained with hematoxylin. Foam cells were counted under a microscope, and the Oil Red O staining was assessed using a color density assay with the iVision software (BioVision Technologies, Exton, PA, USA).

\section{Lipid assay by high-performance liquid chromatography (HPLC)}

To detect lipid deposition in macrophage-derived foam cells, the cellular lipid content, including TC and cholesterol ester (CE), was assessed by HPLC. The cells were rinsed three times with PBS followed by lysis with $0.9 \% \mathrm{NaOH}$ solution. After being homogenized on ice for
$10 \mathrm{~s}$, the BCA kit was used to determine the total protein concentration. Subsequently, an equal volume of trichloroacetic acid was introduced, followed by centrifugation at $800 \mathrm{~g}$ for $10 \mathrm{~min}$. Masterol was used to construct a standard curve. All of these procedures were repeated three times. The samples were dissolved in $100 \mu \mathrm{l}$ of isopropanol-acetonitrile (v/v, 20:80) and then incubated in an ultrasound water bath for $5 \mathrm{~min}$. Ultimately, the samples were analyzed with an Agilent 1100 series HPLC (Agilent Technology, Palo Alto, CA, USA). Lipid accumulation levels are presented as the ratio of CE to TC.

\section{Triglyceride measurements}

The triglyceride content in the cells was determined quantitatively using enzymatic colorimetric assays with kits from Wako (Richmond, VA, USA) according to the manufacturer's protocols. Briefly, approximately $3 \mu \mathrm{l}$ of each sample (cell lysate), standard (cholesterol $200 \mathrm{mg} \mathrm{dl}^{-1}$ ) and blank (distilled water) were added to the prelabeled 
a
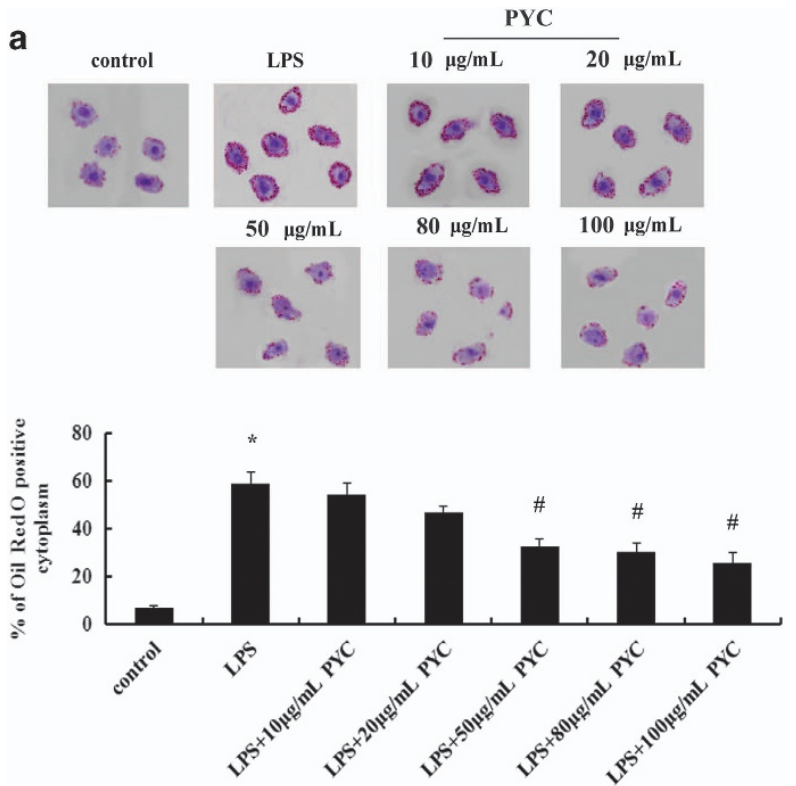

b
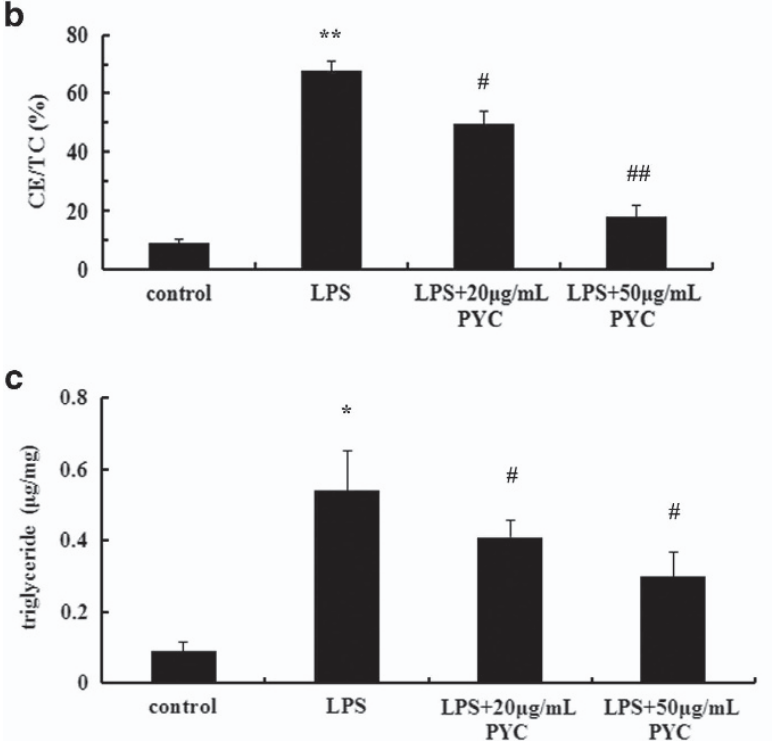

Figure 2 PYC abrogated LPS-induced foam cell formation. Human THP-1 monocytes were stimulated with the indicated doses of PYC prior to incubation with $10 \mu \mathrm{g} \mathrm{ml}^{-1}$ LPS. After exposure to oxidized LDL (CuoxLDL) for $10 \mathrm{~h}$, Oil Red 0 staining was used to label the foam cells (a). The ratio CE/TC was introduced to assess the lipid accumulation (b). The triglyceride content in cells was detected by enzymatic colorimetric assays (c). ${ }^{*} P<0.01$ versus control group; ${ }^{\#} P<0.05$ versus LPS-pretreated cells. ${ }^{\#} P<0.01$.

tubes, followed by the mixing with $2 \mathrm{ml}$ color reagent. After a 5-min incubation at $37^{\circ} \mathrm{C}$, the absorbance was measured at $600 \mathrm{~nm}$.

\section{RNA extraction and real-time PCR}

To obtain total RNA from human THP-1 monocytes, we used the RNAiso Plus Kit (Roche Diagnostics, Mannheim, Germany). The isolated RNA (up to $4 \mu \mathrm{g}$ ) was then reverse-transcribed to synthesize first-strand cDNA with the oligo $(\mathrm{dT})_{18}$ primer using a cDNA Synthesis Kit (Fermentas, St Leon-Rot, Germany). Next, $3 \mu$ of cDNA was subjected to real-time PCR in $20 \mu$ reaction system consisting of $0.4 \mu \mathrm{l} 10 \mu \mathrm{moll}^{-1}$ specific primers, $2 \mu \mathrm{l} \mathrm{cDNA}$ and $\mathrm{H}_{2} \mathrm{O}$. Specific primers for ADRP and ALBP were designed using the IDT DNA website (http://www.idtdna.com): ALBP, 5'-GCCAGGAATTTGACG AAGTCAC-3' and 5'-TTCTGCACATGTACCAGGACAC-3'; and ADRP, 5'-GCACTCACCAAATCAGAGCTG-3' and 5'-AACTTGGC TTCTGAACCAG-3'. All of the primers were synthesized by the TaKaRa Company (Dalian, China) and diluted to a final concentration of $10 \mu \mathrm{M}$ with RNase-Free Water. The reaction conditions were performed according to the instructions provided with the SYBR Premix Ex Taq II Kit (Takara Bio Inc., Otsu, Japan). All of the results are shown as relative mRNA expression data, and the mRNA levels were normalized to $\beta$-actin. All of the samples were assessed in triplicate.

\section{Western blotting}

Following three rinses with PBS, the cells were lysed with RIPA lysis buffer (Beyotime, Nantong, China) to extract the total protein. The protein concentrations were determined using the BCA assay (Pierce, Rockford, IL, USA). For the western blotting analysis, $100 \mu \mathrm{g}$ protein was electrophoresed by sodium dodecyl sulfate-polyacrylamide gel electrophoresis and then transferred onto a polyvinylidene difluoride membrane (Millipore, Beijing, China) in a semi-dry transblot apparatus. After treatment with buffer containing 5\% nonfat dry milk in TBST at $4{ }^{\circ} \mathrm{C}$ overnight, the membrane was incubated with primary antibodies for $1 \mathrm{~h}$ followed by horseradish peroxidase-conjugated secondary antibodies for $1 \mathrm{~h}$. After four washes with TBST buffer, the bound antibodies were visualized using LumiGLo reagent (KPL, Gaithersburg, MD, USA), and the corresponding quantitative analysis of the proteins was performed using Image-Pro Plus 6.0 (Media Cybernetics, Inc., Bethesda, MD, USA). $\beta$-Actin was used for normalization.

\section{Data analysis}

SPSS 11.0 (SPSS Inc., Chicago, IL, USA) was used to analyze the data. All of the results are presented as the mean \pm s.e.m. A typical image from at least three similar experiments is presented. The statistical significance of differences was determined using an independent Student's $t$-test. $P<0.05$ was considered statistically significant.

\section{RESULTS}

Effect of PYC on atherosclerotic plaques, macrophages and lipid levels

To evaluate the role of PYC in the development of atherosclerosis, we constructed mouse models of atherosclerosis. The plaque areas were significantly decreased after 12 weeks with PYC compared with the control groups, indicating that PYC has a protective antiatherogenic effect (Figure 1a). After treatment with LPS and feeding with a high-fat diet, numerous macrophages were observed in the atherosclerotic plaques by immunohistochemical analysis of the macrophage antigen (MOMA)-2. However, PYC treatment resulted in little reduction of the macrophage accumulation in plaques $(P>0.05$; Figure $1 \mathrm{~b})$. Importantly, PYC treatment dramatically decreased the lipid levels in plaques (Figures $1 \mathrm{c}$ and d). Further analysis confirmed that the serum levels of TC, triglycerides and LDL-C were lower in the PYC-treated groups than in the control groups (Figure 1e). Moreover, the levels of HDL-C were upregulated after PYC treatment. Together, these 

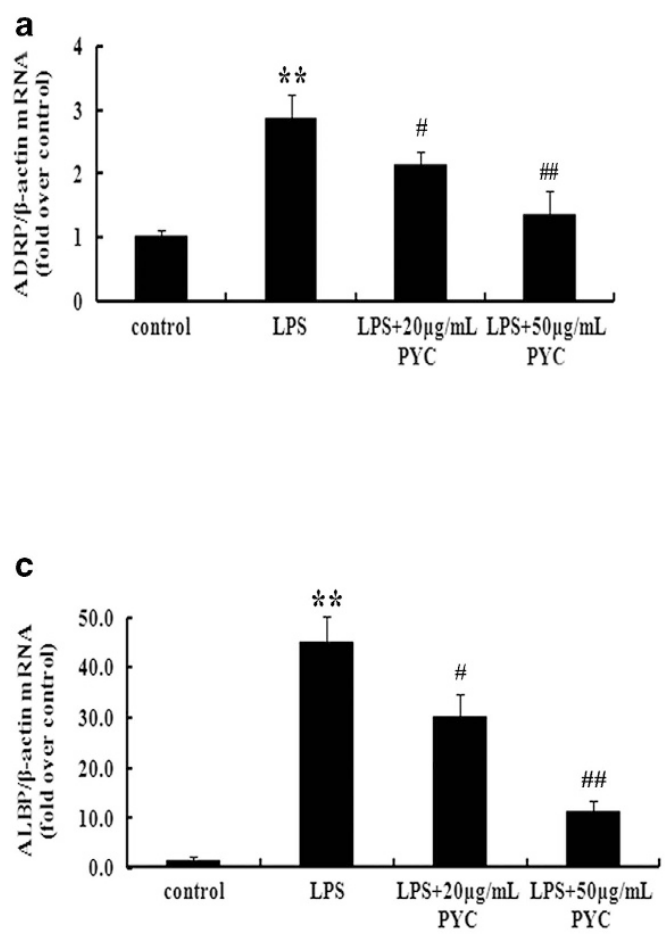
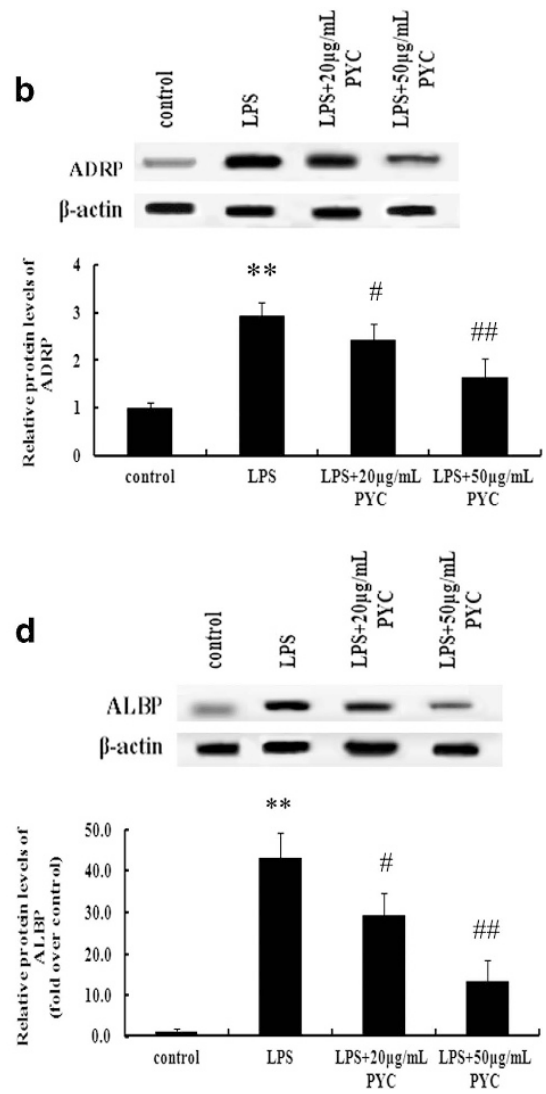

Figure 3 PYC suppressed LPS-mediated ADRP and ALBP expression. Cells were pretreated with the indicated doses of PYC for $8 \mathrm{~h}$, and the mRNA and protein levels of ADRP were determined in LPS-induced THP-1 cells by real-time PCR (a) and western blotting analysis (b). The similar effects of PYC on ALBP mRNA (c) and protein levels (d) were also analyzed. $\beta$-Actin was used for normalization. ${ }^{* *} P<0.01$. $\# P<0.05$. \#\# $P<0.01$.

results suggested that PYC might attenuate the progression of atherosclerosis by regulating lipid levels.

\section{PYC inhibited LPS-induced foam cell formation}

To further clarify the mechanism underlying the PYC-induced antiatherosclerotic effect, we assessed the effect of PYC on lipid-laden foam cell formation in vitro based on its important role in early atherosclerotic lesion development. It is known that THP-1 monocytes can differentiate into macrophages in response to various stimulants, such as LPS. In the present study, a preliminary experiment was performed to explore the effect of different PYC concentrations $(0,10,20,50,80$, $100 \mu \mathrm{g} \mathrm{ml}^{-1}$ ) on cells. Here treatment with LPS significantly induced lipid-laden macrophage-derived foam cell formation compared with the control group based on Oil Red O staining (Figure 2a). Pretreatment with $10 \mu \mathrm{g} \mathrm{ml}^{-1}$ PYC resulted in few differences in macrophage-derived foam cell numbers compared with the LPS group. Stimulation with $20 \mu \mathrm{g} \mathrm{ml}^{-1}$ PYC induced less macrophage-derived foam cell formation compared with the LPS group. Furthermore, an obvious inhibitory effect on foam cell formation was demonstrated in cells that were treated with $50 \mu \mathrm{g} \mathrm{ml}^{-1}$ PYC compared with LPS, resulting in a decrease of approximately one half of the LPS-induced foam cell formation. There were no significant differences between the 50 and the 80 or $100 \mu \mathrm{g} \mathrm{ml}^{-1}$ group (data not shown). Therefore, we chose the representative 20 and $50 \mu \mathrm{g} \mathrm{ml}^{-1}$ concentrations of PYC to perform subsequent experiments. The similar effect of PYC on lipid deposition was further confirmed by HPLC. After exposure to CuoxLDL, abundant lipid content was found in LPS-activated macrophages, and this content is an important component of the genetic program regulating the conversion of macrophages to foam cells (Figure $2 \mathrm{~b}$ ). The ratio CE/TC gradually decreased from 67.86 to $49.72 \%$ and $19.23 \%$ after treatment with 20 and $50 \mu \mathrm{g} \mathrm{ml}^{-1}$ PYC, respectively. Moreover, further analysis confirmed that PYC clearly attenuated the levels of intracellular triglycerides (Figure 2c), suggesting that PYC reduced LPS-induced lipid deposition in a dose-dependent manner. Taken together, these results indicated that PYC could ameliorate LPS-triggered foam cell formation, which would be beneficial for antiatherosclerotic processes.

PYC abrogated lipid accumulation by downregulating the expression of ADRP and ALBP in LPS-induced macrophages As critical lipid droplet-related proteins, the levels of ADRP and ALBP correlate with foam cell formation and atherosclerosis, and a deficiency of these proteins impairs lipid-laden foam cell formation and provides protection against atherosclerosis. ${ }^{15,17}$ 
a
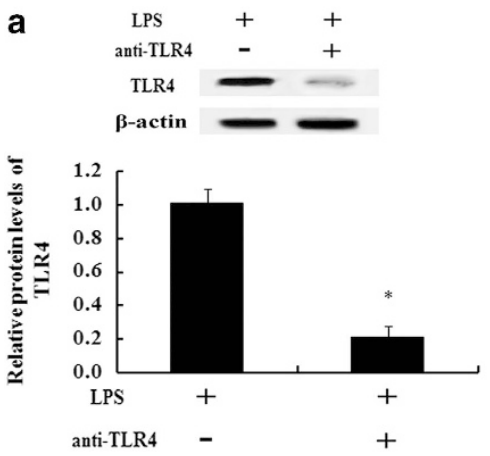

b

$\begin{array}{lll}\text { LPS } & + & + \\ \text { PDTC } & - & +\end{array}$

intra-nuclear p65

$\beta$-actin

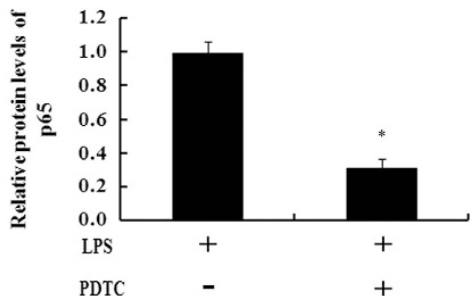

c

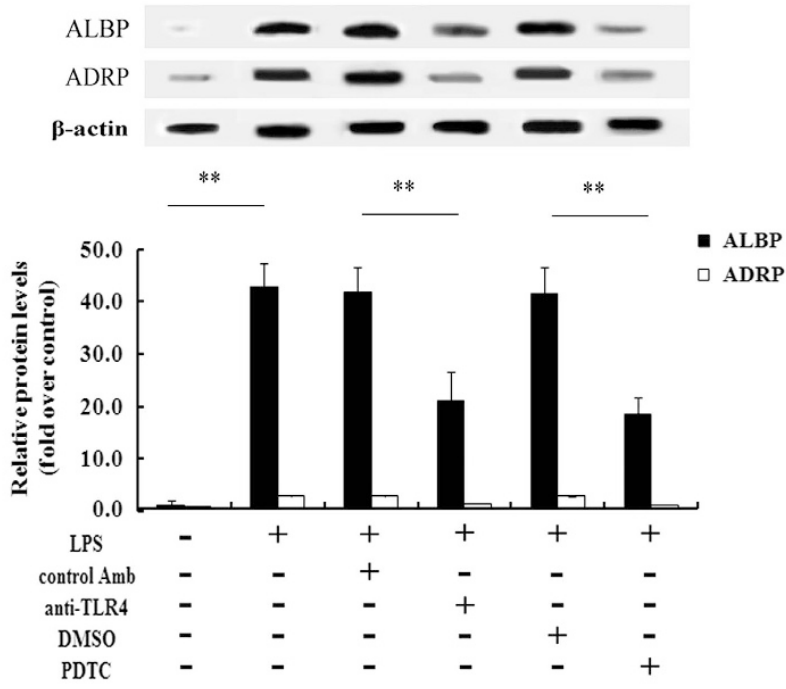

d

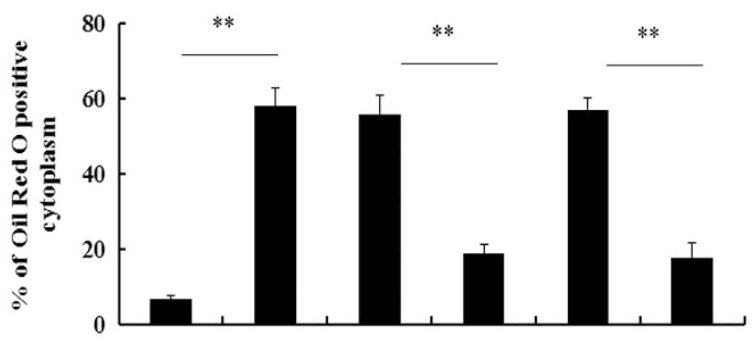

e

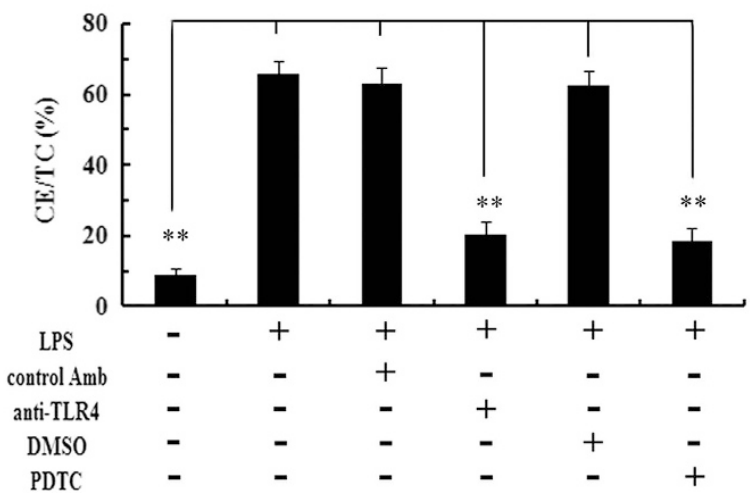

Figure 4 Lipid deposition resulting from ADRP and ALBP upregulation was regulated through the LPS-activated TLR4-NF- $\mathrm{B}$ pathway. Before stimulation with the indicated doses of PYC, THP-1 cells were preconditioned with anti-TLR4 antibody or $25 \mu \mathrm{m}$ PDTC for $2 \mathrm{~h}$, and activation of the TLR4 (a) and NF-KB signaling pathway (b) was analyzed by western blotting analysis. The protein levels of ADRP and ALBP via the LPS-activated TLR4 and NF-kB pathway were determined (c). Corresponding lipid-laden foam cell formation (d) and lipid accumulation (e) was also assessed. ${ }^{*} P<0.05$. ${ }^{*} P<0.01$. 


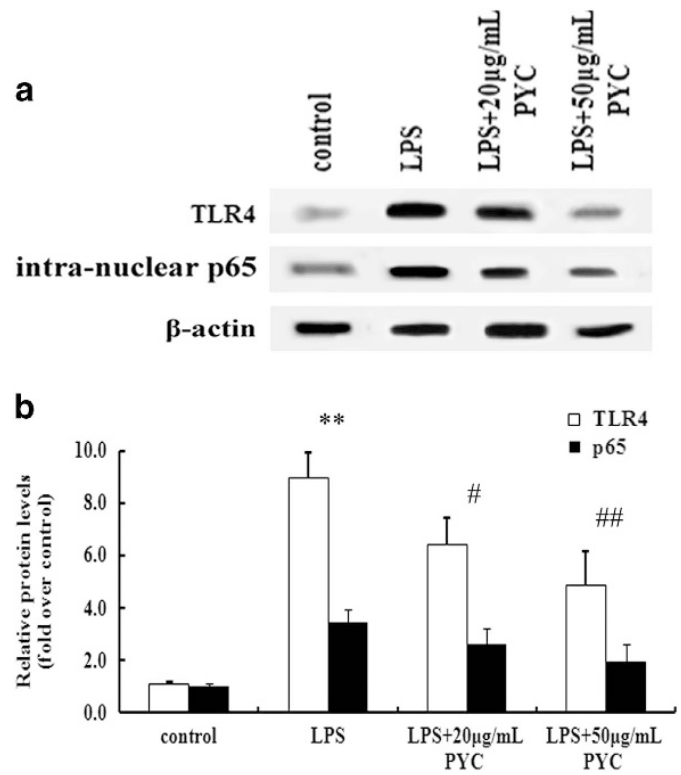

Figure 5 The TLR4-NF-kB pathway accounted for the PYC-induced inhibitory effect on LPS-triggered foam cell formation. Cultured cells were treated with various doses of PYC and then stimulated with $10 \mu \mathrm{g} \mathrm{ml}^{-1}$ LPS for $24 \mathrm{~h}$. After exposure to CuoxLDL, activation of the TLR4 and NF-kB pathway was analyzed by western blotting analysis (a). The corresponding quantified results are also shown (b). ${ }^{* *} P<0.01$ versus control group; ${ }^{\#} P<0.05$ versus LPS-pretreated cells. ${ }^{\#} P<0.01$.

Therefore, we hypothesized that there was an association of PYC-induced foam cell formation with ADRP and ALBP levels. To address this hypothesis, THP-1 cells were treated with the indicated doses of PYC prior to stimulation with LPS. After exposure to PYC, there was an obvious dose-dependent decrease in ADRP mRNA (Figure 3a) and protein levels (Figure 3b) compared with the LPS-treated groups. In addition, PYC induced a significant downregulation of the ALBP mRNA levels as the dose was gradually increased (Figure 3c). Simultaneously, treatment with increased doses of PYC further decreased the LPS-induced expression of ALBP protein (Figure 3d). Based on these results, we concluded that PYC dampened LPS-induced lipid-laden foam cell formation in a dose-dependent manner by downregulating the expression of ADRP and ALBP.

\section{ADRP and ALBP-induced lipid deposition was regulated by} the TLR4-NF-KB pathway in LPS-stimulated THP-1 cells

TLR4, a specific receptor of LPS, is considered to be associated with CVD by contributing to the development of arterial plaques. ${ }^{2,20}$ As a key downstream signaling effector of the TLR4 pathway, NF- $\mathrm{KB}$ is pivotal in the LPS-triggered development of atherosclerosis. ${ }^{20,21}$ To further clarify the molecular mechanism underlying the PYC-induced decrease in the lipid-related proteins ADRP and ALBP, we analyzed the activation of the TLR4-NF-KB pathway. As shown in Figure 4a, pretreatment with specific anti-TLR4 antibody significantly abrogated TLR4 expression. Additionally, preconditioning with PDTC abolished
LPS-induced p65 NF- $\mathrm{kB}$ activation (Figure 4b). Further analysis indicated that pretreatment with the anti-TLR4 antibody caused an obvious inhibition of the LPS-stimulated expression of ADRP and ALBP (Figure 4c). A similar inhibitory effect on ADRP and ALBP expression was confirmed by PDTC preconditioning, concomitant with a striking decrease in lipidladen macrophage foam cell formation based on Oil Red $\mathrm{O}$ staining (Figure 4d) and lipid deposition (Figure 4e). Taken together, these results suggested that ADRP and ALBP were regulated via activation of the TLR4-NF- $\mathrm{KB}$ signaling pathway.

TLR4-NF-кB signaling was a target for the inhibitory effect of PYC on foam cell formation

To further elucidate the molecular mechanism underlying the PYC-induced reduction of foam cell formation, we assessed the correlation between TLR4-NF-KB signaling and PYC. As shown in Figure 5, treatment with $20 \mu \mathrm{g} \mathrm{ml}^{-1}$ PYC strikingly inhibited the activation of TLR4 and its downstream effector NF- $\mathrm{KB}$. When the concentration of PYC reached $50 \mu \mathrm{g} \mathrm{ml}^{-1}$, a more marked inhibitory effect on TLR4-NF- $\mathrm{KB}$ signaling was observed, which indicated that TLR4-NF- $\mathrm{kB}$ signaling was responsible for the effect of PYC on foam cell formation.

\section{DISCUSSION}

In the present study, we first explored the protective effect of PYC against the development of atherosclerosis. Treatment with PYC clearly reduced the areas of plaque induced by LPS in atherosclerotic mice. Further analysis confirmed that PYC treatment had only a marginal effect on the numbers of macrophages but greatly attenuated lipid deposition in the atherosclerotic plaques. An inverse association between HDL-C and an increased risk of CVD has been well established in epidemiological and clinical studies. ${ }^{12}$ Increased HDL-C has potentially antiatherogenic effects. LDL-C represents the amount of cholesterol contained in LDL and is commonly used to estimate the levels of LDLs that are driving the progression of atherosclerosis. ${ }^{22}$ LDL-C has recently been recognized as a potential target to reduce the risk of cardiovascular events. Further analyses indicated that treatment with PYC decreased TC, triglycerides and LDL-C and increased HDL-C levels. Accordingly, our results confirmed a potential protective antiatherosclerotic effect of PYC via the regulation of lipid levels. However, the mechanism by which PYC decreases these lipids remains unknown and requires further exploration.

A hallmark of atherosclerosis is the formation of fatty streaks encompassing the accumulation of lipid-laden macrophages named foam CE cells. Following the uncontrolled uptake of CuoxLDL by macrophages, large amounts of triglycerides are deposited in lipid droplets. Many molecules have been shown to be located on the surface of lipid droplets. Among these, the proteins ADRP and ALBP are considered to be essential for lipid storage, synthesis and metabolism. ${ }^{23,24}$ The overexpression of ADRP and ALBP facilitates macrophage-derived foam cell formation, and this process is blocked by inhibiting the expression of ADRP. ${ }^{14,15,17}$ A previous study has demonstrated that PYC can significantly decrease the expression of ALBP. ${ }^{18}$ 
We have previously demonstrated that PYC downregulates lipid deposition in areas of atherosclerotic lesions. To further investigate the protective effects of PYC against atherosclerosis via the regulation of lipid levels, we explored its impact on lipid droplet formation in vitro. In the present study, LPS clearly accelerated CuoxLDL uptake by THP-1-derived macrophages, and higher $\mathrm{CE} / \mathrm{TC}$ ratios, intracellular triglyceride levels and foam cell numbers were confirmed compared with the control group. Following stimulation with the indicated doses of PYC, we demonstrated a dose-dependent decrease in foam cell formation as well as lipid deposition compared with the LPS-treated group. Further mechanistic analysis suggested that PYC significantly decreased the mRNA and protein levels of LPS-stimulated ADRP and ALBP, followed by a decrease in lipid accumulation. Based on the key function of ADRP and ALBP in foam cell formation, we concluded that PYC inhibited LPS-induced lipid accumulation by downregulating the expression of ADRP and ALBP, which may provide a beneficial antiatherosclerotic effect in CVDs.

It is known that TLR4 is a specific receptor for LPS, and the absence of TLR4 diminishes atherosclerotic plaque formation. ${ }^{25,26} \mathrm{NF}-\mathrm{\kappa B}$ is known to be a critical downstream effector, and the blockade of LPS-induced NF- $\mathrm{KB}$ signaling reduces atherosclerosis. ${ }^{27}$ It has been confirmed that LPS significantly increases ADRP and ALBP expression. ${ }^{12,28}$ Therefore, we hypothesized that there was a relationship between the TLR4-NF-KB pathway and the expression of ADRP and ALBP. Consistent with a previous study, LPS upregulated the mRNA and protein levels of ADRP and ALBP together with increased lipid deposition. Following pretreatment with specific antiTLR4 antibody or the NF- $\mathrm{kB}$ inhibitor PDTC, this increase was reduced, which suggested that TLR4-NF- $\mathrm{KB}$ signaling was responsible for the LPS-induced upregulation of ADRP and ALBP expression during foam cell formation. The PYCinduced downregulation of ADRP and ALBP expression would demonstrate its association with the TLR4-NF- $\mathrm{KB}$ pathway. Consistent with our hypothesis, preconditioning with PYC dramatically impeded TLR4 expression as well as NF- $\kappa B$ activation triggered by LPS, indicating that PYC strongly mitigated LPS-initiated ADRP and ALBP expression through the TLR4-NF-kB pathway. However, the mechanism by which PYC exerts its inhibitory on TLR4-NF- $\mathrm{KB}$ has not been described, and whether other signaling mechanisms are also involved in this progress remains unknown. These questions will be explored in our future studies.

The present findings may provide potential insight into the antiatherosclerotic effect of PYC via the mediation of lipid levels, suggesting that PYC is a promising therapeutic agent against atherosclerotic CVDs. However, the antiatherosclerotic effects of PYC by regulating lipid levels were demonstrated in vitro, and direct evidence for the antiatherosclerotic functions of PYC through the modulation of ADRP and ALBP expression via the TLR4-NF- $\mathrm{KB}$ pathway in vivo remains to be demonstrated. Previous research has confirmed that ApoE - / - mice lacking TLR4 exhibit reduced plaque lipid content, macrophage numbers and pro-inflammatory cytokine levels, suggesting a pathophysiological link between immunity and atherosclerosis. ${ }^{29}$ However, the present study demonstrated that PYC treatment caused a decrease in the number of macrophages that accumulated in plaques, but this result was not significantly different from the control groups. The effects of a drug in vivo are known to be complicated and affected by various factors, such as the dose, timing, drug delivery technique and other factors that are present in vivo. Perhaps the dose of PYC used in the present study only affected TLR4-mediated lipid metabolism but had little effect on macrophage recruitment. Higher doses of PYC or other routes of drug delivery will be assessed in future studies to explore the effect of this compound on macrophage recruitment. Furthermore, whether PYC affects macrophage migration to damaged endothelial cells via the TLR4 pathway in vivo necessitates further investigation.

Bacterial infection has been confirmed to be significantly associated with the development of atherosclerosis and its clinical complications, including unstable angina, myocardial infarction and stroke. ${ }^{30}$ Here we constructed LPS and high-fat diet-induced atherosclerotic mice. The results of this study aid in clarifying the mechanism by which PYC attenuates bacterial infection-triggered pathological processes associated with atherosclerosis. PYC is known to have potential protective anti-inflammatory properties. ${ }^{3,31}$ Whether PYC can attenuate the development of atherosclerosis by regulating the inflammatory response remains unclear. The mechanisms underlying the effects of PYC on the progression of atherosclerosis remain to be validated in future investigations.

\section{CONFLICT OF INTEREST}

The authors declare no conflict of interest.

1 Hansson GK. Inflammation, atherosclerosis, and coronary artery disease. N Engl J Med 2005; 352: 1685-1695.

2 Hansson GK, Robertson A-KL, Söderberg-Nauclér C. Inflammation and atherosclerosis. Annu Rev Pathol Mech Dis 2006; 1: 297-329.

3 McCullough ML, Peterson JJ, Patel R, Jacques PF, Shah R, Dwyer JT. Flavonoid intake and cardiovascular disease mortality in a prospective cohort of US adults. Am J Clin Nutr 2012; 95: 454-464.

4 Mulvihill EE, Huff MW. Protection from metabolic dysregulation, obesity, and atherosclerosis by citrus flavonoids: activation of hepatic PGC1 $\alpha$-mediated fatty acid oxidation. PPAR Res 2012; 2012: 857142.

5 Zibadi S, Rohdewald PJ, Park D, Watson RR. Reduction of cardiovascular risk factors in subjects with type 2 diabetes by Pycnogenol supplementation. Nutr Res 2008; 28: 315-320.

6 Watson R. Reduction of cardiovascular disease risk factors by French maritime pine bark extract. Cardiovasc Rev Rep 1999; 20: 326-329.

7 Yamamoto K, Ando J. New molecular mechanisms for cardiovascular disease:blood flow sensing mechanism in vascular endothelial cells. J Pharmacol Sci 2011; 116: 323-331.

8 Favero G, Paganelli C, Buffoli B, Rodella LF, Rezzani R. Endothelium and its alterations in cardiovascular diseases: life style intervention. Biomed Res Int 2014; 2014: 801896.

9 Mehta JL, Basnakian AG. Interaction of carbamylated LDL with LOX-1 in the induction of endothelial dysfunction and atherosclerosis. Eur Heart $J$ 2014; 35: 2996-2997.

10 Enseleit F, Sudano I, Périat D, Winnik S, Wolfrum M, Flammer AJ et al. Effects of Pycnogenol on endothelial function in patients with stable coronary artery disease: a double-blind, randomized, placebo-controlled, cross-over study. Eur Heart J 2012; 33: 1589-1597. 
11 Feng $X$. Natural IgM specific for oxLDL protects murine macrophages from foam cells formation but lipopolysaccharide (LPS) disrupts the course by up-regulating the expression of $\mathrm{Fc} \alpha / \mu$ receptor. Heart 2011; 97: A58-A58.

12 Cai Y, Li J-D, Yan C. Vinpocetine attenuates lipid accumulation and atherosclerosis formation. Biochem Biophys Res Commun 2013; 434: 439-443.

13 Chapman KD, Dyer JM, Mullen RT. Biogenesis and functions of lipid droplets in plants: Thematic Review Series: Lipid Droplet Synthesis and Metabolism: from Yeast to Man. J Lipid Res 2012; 53: 215-226.

14 Imamura M, Inoguchi T, Ikuyama S, Taniguchi S, Kobayashi K, Nakashima $\mathrm{N}$ et al. ADRP stimulates lipid accumulation and lipid droplet formation in murine fibroblasts. Am J Physiol Endocrinol Metab 2002; 283: E775-E783.

15 Paul A, Chang BH-J LiL, Yechoor VK, Chan L. Deficiency of adipose differentiation-related protein impairs foam cell formation and protects against atherosclerosis. Circ Res 2008; 102: 1492-1501.

16 Kazemi MR, McDonald CM, Shigenaga JK, Grunfeld C, Feingold KR. Adipocyte fatty acid-binding protein expression and lipid accumulation are increased during activation of murine macrophages by toll-like receptor agonists. Arterioscler Thromb Vasc Biol 2005; 25: 1220-1224.

17 Fu Y, Luo N, Lopes-Virella MF, Garvey WT. The adipocyte lipid binding protein (ALBP/aP2) gene facilitates foam cell formation in human THP-1 macrophages. Atherosclerosis 2002; 165: 259-269.

18 Gu J-Q, Ikuyama S, Wei P, Fan B, Oyama J-i, Inoguchi T et al. Pycnogenol, an extract from French maritime pine, suppresses Toll-like receptor 4-mediated expression of adipose differentiation-related protein in macrophages. Am J Physiol Endocrinol Metab 2008; 295: E1390-E1400.

$19 \mathrm{Li} \mathrm{R}$, Chen B, Wu W, Bao L, Li J, Qi R. Ginkgolide B suppresses intercellular adhesion molecule-1 expression via blocking nuclear factor$\mathrm{\kappa b}$ activation in human vascular endothelial cells stimulated by oxidized low-density lipoprotein. J Pharmacol Sci 2009; 110: 362-369.

20 den Dekker WK, Cheng C, Pasterkamp G. Duckers HJ. Toll like receptor 4 in atherosclerosis and plaque destabilization. Atherosclerosis 2010; 209: 314-320.

21 Gareus R, Kotsaki E, Xanthoulea S, van der Made I, Gijbels MJ, Kardakaris $\mathrm{R}$ et al. Endothelial cell-specific NF-kB inhibition protects mice from atherosclerosis. Cell Metab 2008; 8: 372-383.

22 Pankow JS, Boerwinkle E, Adams PC, Guallar E, Leiendecker-Foster C, Rogowski J et al. HFE C282Y homozygotes have reduced low-density lipoprotein cholesterol: the Atherosclerosis Risk in Communities (ARIC) Study. Transl Res 2008; 152: 3-10.
23 Sparks LM, Moro C, Ukropcova B, Bajpeyi S, Civitarese AE, Hulver MW et al. Remodeling lipid metabolism and improving insulin responsiveness in human primary myotubes. PLOS ONE 2011; 6: e21068.

24 Fu Y, Luo L, Luo N, Garvey WT. Lipid metabolism mediated by adipocyte lipid binding protein (ALBP/aP2) gene expression in human THP-1 macrophages. Atherosclerosis 2006; 188: 102-111.

25 Lu Z, Zhang X, Li Y, Jin J, Huang Y. TLR4 antagonist reduces early-stage atherosclerosis in diabetic apolipoprotein E-deficient mice. J Endocrinol 2013; 216: 61-71.

26 Michelsen KS, Doherty TM, Shah PK, Arditi M. Role of Toll-like receptors in atherosclerosis. Circulation Res 2004; 95: e96-e97.

27 Kim S, Park J, Kim K, Lee W, Kim K, Park K. Melittin inhibits atherosclerosis in LPS/high-fat treated mice through atheroprotective actions. J Atheroscler Thromb 2011; 18: 1117-1126.

28 Feingold KR, Kazemi MR, Magra AL, McDonald CM, Chui LG, Shigenaga JK et al. ADRP/ADFP and Mal1 expression are increased in macrophages treated with TLR agonists. Atherosclerosis 2010; 209 : 81-88.

29 Michelsen KS, Wong MH, Shah PK, Zhang W, Yano J, Doherty TM et al. Lack of Toll-like receptor 4 or myeloid differentiation factor 88 reduces atherosclerosis and alters plaque phenotype in mice deficient in apolipoprotein E. Proc Natl Acad Sci USA 2004; 101: 10679-10684.

30 Muhlestein JB. Bacterial infections and atherosclerosis. J Investig Med 1998; 46: 396-402.

31 Lee MS, Moon KY, Bae DJ, Park MK, Jang AS. The effects of pycnogenol on antioxidant enzymes in a mouse model of ozone exposure. Korean J Intern Med 2013; 28: 216-223

(c) (1) (5) This work is licensed under a Creative Commons Attribution-NonCommercial-ShareAlike 4.0 International License. The images or other third party material in this article are included in the article's Creative Commons license, unless indicated otherwise in the credit line; if the material is not included under the Creative Commons license, users will need to obtain permission from the license holder to reproduce the material. To view a copy of this license, visit http:// creativecommons.org/licenses/by-nc-sa/4.0/ 